\title{
A Novel Method VESTAL to Label Lumber Vertebrae and Intervertebral Discs
}

\author{
Rayudu Srinivas \\ SSAIST \\ Surampalem \\ Andhra Pradesh
}

\author{
K.V. Ramana, Ph.D. \\ Professor and BoS Chairman \\ Computer Science and Engineering \\ JNTUK, Kakinada
}

\begin{abstract}
This paper presents a novel method Vertebrae Statistics description Algorithm (VESTAL) to label lumber vertebrae and intervertebral discs (IVDs). Each vertebra and IVD has certain statistical features and properties. To label vertebrae and IVDs, a new equation to model the path of spinal cord is derived using statistical properties of the spinal canal. VESTAL uses this equation for labelling Lumber vertebrae and IVDs by determining both posterior, interior width and heights. The calculated values are compared with real values which are measured using scale and the comparison produced $95 \%$ efficiency and accuracy in results. The VESTAL is applied on 50 patients, 250 MR images and obtained 96\% accuracy in labelling.
\end{abstract}

\section{Keywords}

Disc, Labelling, Intervertebral Disc, Vertebrae, Spine, Statistics, Texture.

\section{INTRODUCTION}

The second most common neurological ailment in the world after headache is lower back pain [1]. Most of the employees, specifically the youngsters and the middle aged are suffering from neck and lower back pain. Vertebrae and IVDs abnormalities are the major causes of lower back pain and neck pain. Hence, lumbar area and cervical area become hot areas for research. Researchers developed semi-automated and fully-automated methods to detect vertebral abnormality, wedge fractures, bulging and other related problems. Labelling vertebrae and intervertebral discs are very important in the diagnoses of spinal problems. To diagnosis spinal problems, radiologists generally label the MR Images manually. Manual labelling is tedious and error prone. In manual labelling, radiologist labels the vertebrae by identifying one reference point generally and this reference point is sacrum or atlas. Accurate labelling of the vertebrae and IVDs are necessary because the backbone has wide variability including degree of bending of the vertebral column, sizes, shapes and appearances of discs and vertebrae [2-3]. Figure 1 shows a normal and abnormal Lumber spine images.

One key issue of labelling is the design of a Computer Aided Diagnosis (CAD) system for lumbar and cervical area. Labelling and segmentation are challenging task for developing fully automated models because the CT and MR images can be extremely inhomogeneous, neighbouring irrelevant structures can be connected and might have similar intensities. Computer-Aided Diagnosis aims at assisting medical doctors in their diagnostic decision-making process. Even though the final diagnosis should be made by the doctor, a CAD system has been widely utilized based on a variety of modalities such as CT and MRI, assisting physicians in their early detection of various abnormalities such as breast cancer, lung nodules, vertebral fractures and intracranial aneurysms [4]. T1 and T2 images are frequently used in diagnosis of the spinal images because they provide better contrast between tissues. As a result this study focuses on MR image processing for intervertebral disc degeneration in spinal diseases.
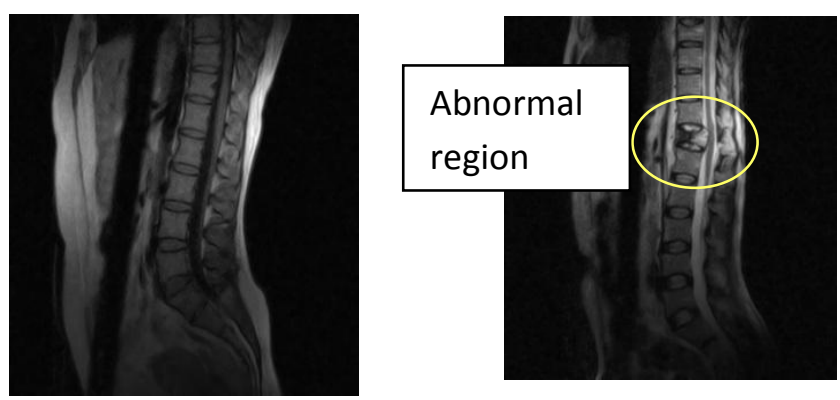

Fig. 1 a) Normal MR Image b) Degenerated vertebra MR images

In literature most of the authors proposed methods based on reference points or MR Images in standard position [5-7]. The selection of reference point plays a key role in labelling and it depends on the expertise of radiologist. Many researchers have investigated the problem of labelling the anatomic structures of the vertebral column. However, these studies are limited by their data set size and thus limit the significance of the validation. The proposed method is based on statistical properties hence named as vertebrae statistic description algorithm (i.e. VESTAL) does not require such reference point and the image need not be in standard position, it can be in any orientation. VESTAL uses anterior, posterior length and width of Lumber vertebrae and based on the relations it labels vertebrae and IVDs.

The proposed VESTAL is also useful for detecting the wedge fractures. Two variants of VESTAL namely semi-automated and fully-automated algorithms are proposed based on seed point selection. In semi-automated method a seed pixel is manually specified. In fully-automated method, a seed pixel is selected based on the dimensions of the image. Starting from the initial seed point a progressive adaptation algorithm is applied to trace the spinal canal. VESTAL performs the following major tasks.
a. Identify spinal canal
b. Tracing the vertebrae
c. Calculate four corner points for each vertebra
d. Recognition of vertebrae center line
e. Localization of the intervertebral disks
f. Labelling Lumber vertebrae and IVDs 
The rest of the paper is organized as follows: the related work is presented in section 2. Proposed methodology in detail is given in section 3 . Results are presented in section 4. Finally concluding remarks are given in section 5 .

\section{RELATED WORK}

Mohammed Benjelloun et al. proposed a semi-automated model called ASM model to detect vertebrae shape [8]. They prepared a model shape of the vertebrae by considering vertebrae features like length and width. Seifert et al. proposed a knowledge-based approach for cervical spine that combines object recognition, segmentation algorithms and anatomical knowledge for the spine reconstruction from MR images [5].

Ayse Betul Oktay et al. [6] presented a method for localizing and labelling the lumbar vertebrae and intervertebral discs in mid-sagittal MR image slices. Their approach is based on a Markov-chain-like graphical model of the ordered discs and vertebrae in the lumbar spine. Ruiqiong Shi et al. [7] proposed semi-automatic method to detect disc in spine. In preprocessing, average values for window, vertebrae length and width are calculated. To detect the discs from complete spine of 1.5T MRI images are taken as input.

Intensity profiles are used by Peng et al. [9] to localize the 24 articulated vertebrae. Huang et al. proposed a method to [4] detect and segment vertebra with wavelet transform-based Adboost and iterative normalized cut. Schmidt et al. [10] established a probabilistic inference method that deals with the possible locations of the spinal discs in 3D MR Images. This approach uses a part-based model that describes the disc appearances by employing a tree classifier. An Hough Transform (HT) based method to localize discs from video fluoroscopic CT images is proposed by Zheng et al. [11]. Due to the likeness of the discs and local character of the HT algorithm, their system needs to utilize human vision to recommend approximate disc positions. J.J Corso et al. [12], used a graphical model for the lumbar disc localization.

Major et al. presented an automated system for land marking and labelling spinal columns in 3D CT datasets. They designed a framework with two goals in mind. First, they relaxed input data requirements found in the literature and labelled both full and partial spine scans. Secondly, they intended to fulfill the performance requirement for daily clinical use and developed a high throughput system capable of processing thousands of slices in just a few minutes [13].

Tunset et al. measured width and height of the vertebrae and intervertebral disc by magnifying MR image by 1100 to 1200 times to get accurate results and to minimize the error. Measurements were performed on MRIs from 16 people with and 16 people without lumbar disc herniation, purposefully chosen to represent all possible disc contours among participants in a general population study cohort [14]. In literature, authors proposed image analysis methods to study about spinal column including vertebra and disc detection, segmentation and labelling by considering reference point [15-18]. To overcome such drawbacks a novel method VESTAL is proposed.

\section{METHODOLOGY}

The proposed model VESTAL labels vertebrae and IVDs based on statistical properties of spinal canal, vertebrae and IVDs. The architecture of the proposed model is shown in figure 2 . The phases of the architecture are

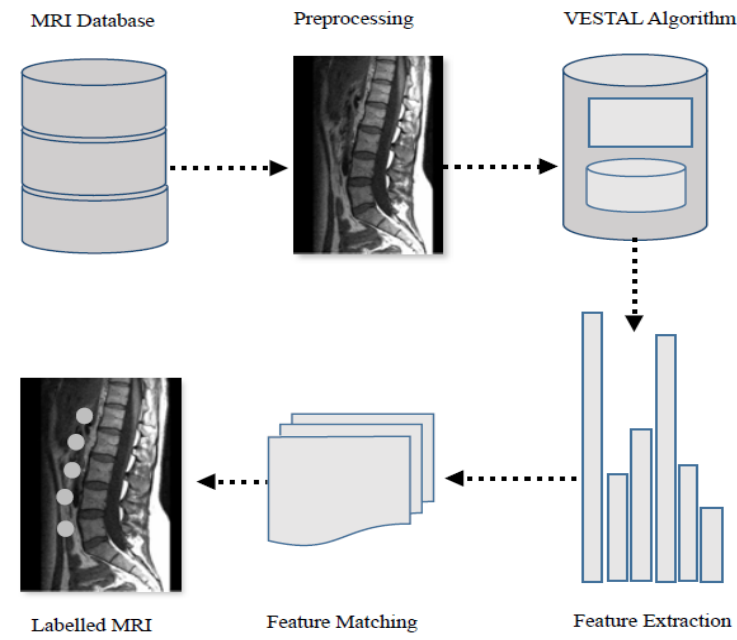

Fig. 2: Architecture of proposed model to label vertebrae and dises

\subsection{Spinal Cord Extraction}

To detect path of spinal cord, Region Of Interest (ROI) is selected in semi-automated method by applying region growing and region splitting algorithms. Once ROI is selected, various operators can be used to detect spine path in MR Image. Mostly these are based on gradient operator. The gradient vector points in the direction of maximum rate of change of at $(\mathrm{x}, \mathrm{y})$ is

$$
\nabla F=\left[\begin{array}{l}
G x \\
G y
\end{array}\right]=\left[\begin{array}{l}
\frac{\partial f}{\partial x} \\
\frac{\partial f}{\partial y}
\end{array}\right]
$$

$$
\begin{aligned}
& \text { Gradient is given as: } \nabla \mathrm{f}=\operatorname{mag}(\nabla \mathrm{F}) \\
& \qquad=\left[\mathrm{G}_{\mathrm{x}}^{2}+\mathrm{G}_{\mathrm{y}}^{2}\right]^{1 / 2} \\
& \text { Direction angle of } \nabla f \text { at }(x, y): a(x, y) \\
& \qquad=\tan ^{-1}\left[\frac{G_{y}}{G_{x}}\right]
\end{aligned}
$$

Sobel operator is applied to detect the edges. Sobel operators provide both a differencing and a smoothing effect. The Sobel operator relies on central differ

$$
\begin{gathered}
G_{x}=\left(Z_{7}+2 Z_{8}+Z_{9}\right) \\
-\left(Z_{1}+2 Z_{2}+Z_{3}\right) \\
G_{y}=\left(Z_{3}+2 Z_{6}+Z_{9}\right) \\
-\left(Z_{1}+2 Z_{4}+Z_{7}\right)
\end{gathered}
$$

The detection of spinal canal as follows: A seed pixel is selected randomly or more meaningfully by using various statistical techniques or by using previous experience. In proposed method central pixel is chosen as seed pixel, using this spinal canal is detected. The spinal canal expression is derived by considering 250 spine images using statistical features. The derived equation has the following generalized form

$$
\begin{gathered}
\mathrm{X}=\mathrm{A}+\mathrm{BY} \mathrm{Y}^{1}+C Y^{2}+D Y^{3} \\
+\cdots
\end{gathered}
$$


Using above equation spinal canal with $95 \%$ accuracy can be detected. If the power considered for equation increases then the efficiency of the spinal cord detection decreases. Better results can be obtained using quadratic equation.

\subsection{Vertebrae Detection}

Once spinal canal is detected the vertebrae can be detected using statistical features. After measuring statistical properties of vertebra and intervertebral disc, the average values, deviation from mean values of vertebrae width, height, disc width and height are calculated using the expression 7 and 8

$$
\begin{aligned}
& \mu=\sum_{1}^{n} \frac{X i}{n} \\
& \sigma^{2}=\frac{\sum(x-\mu)^{2}}{n}
\end{aligned}
$$

\subsection{Intervertebral Disc Search}

After detecting spinal canal, it is easy to detect vertebrae and intervertebral discs. These tissues may be to the left or right of spinal canal based on the orientation of the spine image. Using modified four or eight connected algorithm or using linear search algorithm intervertebral discs can be detected.

//img[][] vector determines the position of vertebra

$/ / p, q$ intensity ranges of the vetebra

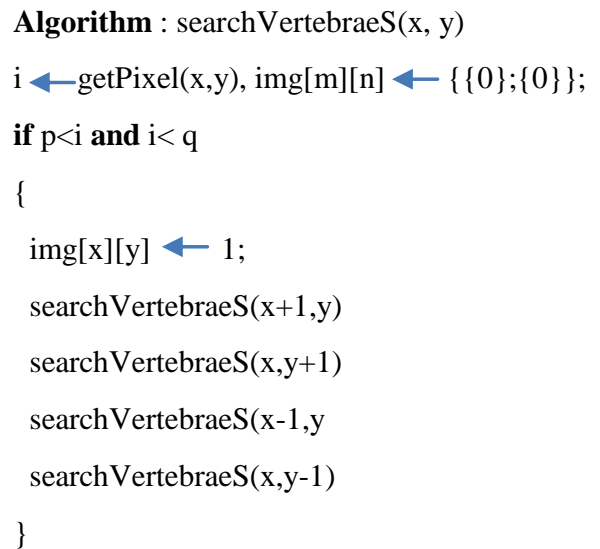

Algorithm : searchVertebraeF()

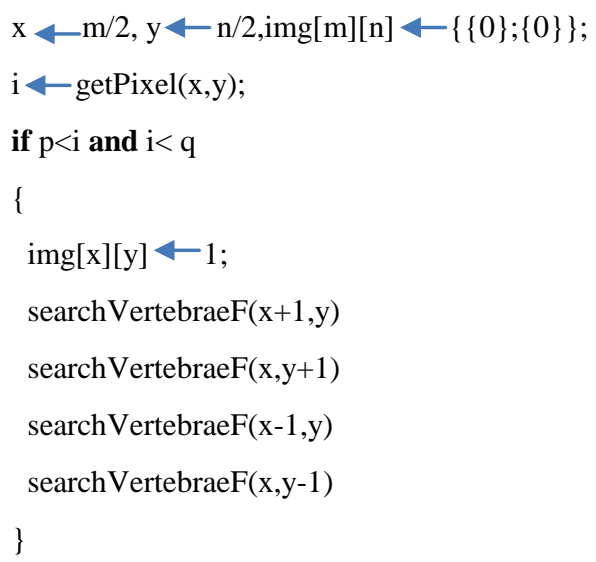

Algorithm: linearSearch()

for $\mathrm{i} \leftarrow 0$ to $\mathrm{m}$ for $\mathrm{j} \leftarrow 0$ to $n$

$$
\text { do }\left\{\begin{array}{l}
\mathrm{k} \leftarrow \operatorname{getPixel}(\mathrm{i}, \mathrm{j}) \\
\text { if } \mathrm{p}<\mathrm{k} \text { and } \mathrm{k}<\mathrm{q} \\
\operatorname{img}[\mathrm{i}][\mathrm{j}] \leftarrow-1 ;
\end{array}\right.
$$

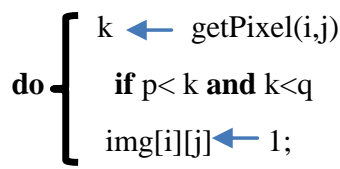

\subsection{Features Detection}

Feature detection and matching are the essential components of many image processing applications. In feature detection phase, features of the image like line end points, corner point edges, center of gravity closed regions contours, closed regions intersection points are detected.

\subsection{Feature Matching}

There are two main approaches for finding feature points and their correspondences. The first step is to find features in one image that can be accurately tracked using a local search technique such as correlation or least squares. In order to extract a shape from an image, it is necessary to identify it from the background elements. This can be done by considering the intensity information or by comparing the pixels against a given template. The second step is Template matching which is a model-based approach in which the shape is extracted by searching for the best correlation between a known model and the pixels in an image. The Hough Transform defines an efficient implementation of template matching for binary templates. This technique is capable of extracting simple shapes such as lines and quadratic forms as well as arbitrary shapes after detecting and determining the features that come from corresponding locations in different images. In this phase, the features detected from test image are used for correspondence between test image and average features extracted from trained images. Here some mathematical expressions mostly translation, rotation, scaling and combination of these methods are used for proper alignment before matching the properties. Once features and their descriptors are extracted from two or more images, the next step is to establish some preliminary feature matches between these images. The equations for these transformations are given as composite transformation.

$\left[\begin{array}{lll}r s_{x x} & r s_{x y} & t r s_{x} \\ r s_{y x} & r s_{y y} & t r s_{y}\end{array}\right]$

Using above approaches the complete procedure to detect vertebrae and IVDs consolidated are given in algorithm.

\subsection{VESTAL Algorithm}

1: Create data base of MRI spine image of uniform size.

2: Sequence of median and Weiner filter followed by image segmentation concepts, Region splitting and Region merging are applied to remove noise and irrelevant parts of the image.

3: Semi-automated or fully-automated methods applied on filtered images to measure statistical features.

4: Extracted features length, width and texture from input test image using step 3.

5: Feature matching algorithm is used to label vertebrae and IVDs.

Semi-automated method to detect vertebra length, width measurements

// Input parameters: LLCx, LLCy, URCx, URCy

// LLC : Lower Left Corner, URC: Upper Right Corner

$/ / \mathrm{p}, \mathrm{q}$ : vertebra intensity range of values

$/ / \mathrm{r}$, s: disc intensity range of values 
$/ / \mathrm{cr}[]$ : control register used to store location of vertebra pixel information

top $\longleftarrow$ URCy, bottom $\leftarrow$ LLCy, j $\leftarrow 0$, cr[] $\leftarrow\{0\}$

while bottom<top

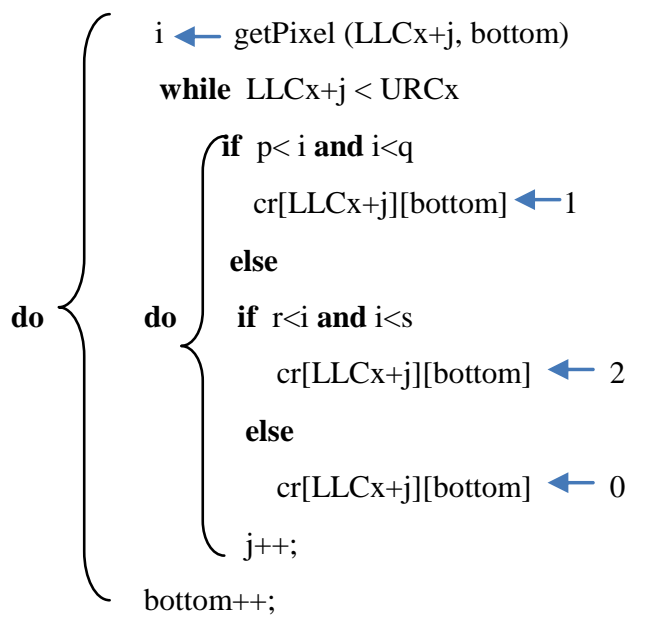

After execution, the control register contains approximate vertebra values. From this control register, values of pixel, other pixels belong to IVD and vertebra are detected using vertebraeSearchS() or vertebraeSearchF() algorithms. While detecting pixel, calculate minimum and maximum values for storing $\mathrm{x}$ and $\mathrm{y}$ coordinates value from which vertical length of vertebra is measured. Once width and length are calculated for all vertebra, a model is prepared for labelling new input test image.

\section{Fully automated method to detect vertebra length, width}

\section{measurements}

// Input parameters: m,n

$/ / \mathrm{mXn}$ : size of image

$/ / \mathrm{p}, \mathrm{q}$ : spinal canal intensity range of values

$/ / \mathrm{cr}[][]$ : control register to store spinal canal pixel coordinate values

$$
\operatorname{cr}[][] \leftarrow\{0\}, \mathrm{x} \leftarrow \mathrm{m} / 2, \mathrm{y} \leftarrow \mathrm{n} / 2, \mathrm{i} \leftarrow \mathrm{x}, \mathrm{j} \leftarrow \mathrm{y}, \text { flag } \leftarrow 0
$$

while $\mathrm{i}>0$

$$
\text { do }\left\{\begin{array}{c}
\mathrm{t} \leftarrow \text { getPixel(i,j) } \\
\text { if } \mathrm{p}<\mathrm{t} \text { and } \mathrm{t}<\mathrm{q} \\
\text { flag } \leftarrow 1 ; \\
\text { break; } \\
\mathrm{i}--
\end{array}\right.
$$

$$
\begin{aligned}
& \text { if }(\text { flag }=0) \\
& \text { do }\left\{\begin{array}{l}
\mathrm{i} \leftarrow \mathrm{m} / 2 \\
\text { while } \mathrm{i}<\mathrm{n}
\end{array}\right. \\
& \text { do }\left\{\begin{array}{l}
\mathrm{t} \leftarrow \text { getPixel }(\mathrm{i}, \mathrm{j}) \\
\text { if } \mathrm{p}<\mathrm{t} \text { and } \mathrm{t}<\mathrm{q} \\
\text { flag } \leftarrow 1 \text {; break } \\
\mathrm{i}++;
\end{array}\right.
\end{aligned}
$$

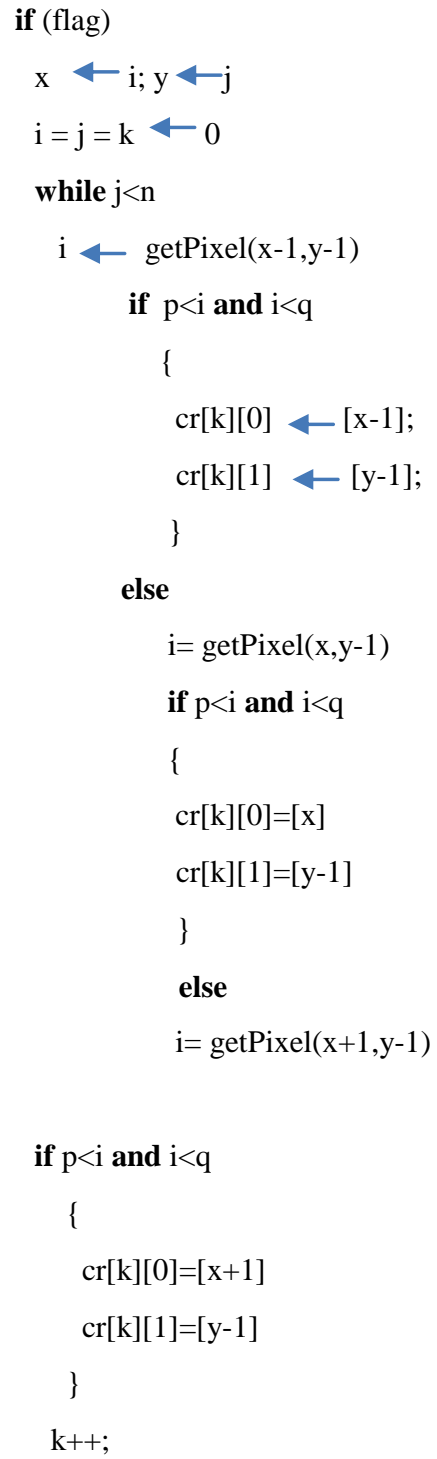

The status register contains approximate spine path using this information vertebrae and IVDs can be located.

\section{RESULTS}

MR Images are magnified to 2 to 4 times for accurate measurements. The coordinates $\mathrm{X}$ and $\mathrm{Y}$ are used to measure width and height respectively. Table 1 contains average values of width and height of vertebrae measured manually from MRI. The values are measured by considering ROI in MR image. Figure 3 shows the relationship between $\mathrm{X}$ and $\mathrm{Y}$ (i.e. $X$ and $f(x)$ ) coordinates drawn using proposed algorithm.

Vertebrae and IVDs locations are detected using this shape of the spine. The located vertebrae width and height average values are measured and these dimensions are scaled to have uniform values and these values are used to plot graphs. The output of sobel edge detection and proposed spine canal detection are shown in figure 4.

The relationship between vertebra anterior, posterior width and height of Lumber vertebrae L1 to L5 is given in figure 5 . Figures 6 to 10 shows the relation between individual Lumber vertebra's anterior and posterior width and height. The label A specifies anterior width of vertebrae, a1 and a2 specifies the range of this width. The label $\mathrm{C}$ specifies posterior width of Lumber vertebrae, $\mathrm{c} 1$ and $\mathrm{c} 2$ specifies the range of this width. The label $\mathrm{B}$ anterior height of Lumber 
vertebrae, b1 and b2 specifies the range of this height. The label D posterior height Lumber vertebrae, $\mathrm{d} 1$ and $\mathrm{d} 2$ specifies the range of this height.

Table 1 : Spinal coordinate values

\begin{tabular}{|c|c|c|}
\hline S.NO & Y & $X$ \\
\hline 1 & 3.5 & 9 \\
\hline 2 & 3.55 & 8.5 \\
\hline 3 & 3.6 & 8 \\
\hline 4 & 3.6 & 7.5 \\
\hline 5 & 3.65 & 7 \\
\hline 6 & 3.65 & 6.5 \\
\hline 7 & 3.65 & 6 \\
\hline 8 & 3.65 & 5.5 \\
\hline 9 & 3.6 & 5 \\
\hline 10 & 3.6 & 4.5 \\
\hline 11 & 3.5 & 4 \\
\hline 12 & 3.45 & 3.5 \\
\hline 13 & 3.35 & 3 \\
\hline 14 & 3.3 & 2.5 \\
\hline 15 & 3.3 & 2 \\
\hline
\end{tabular}

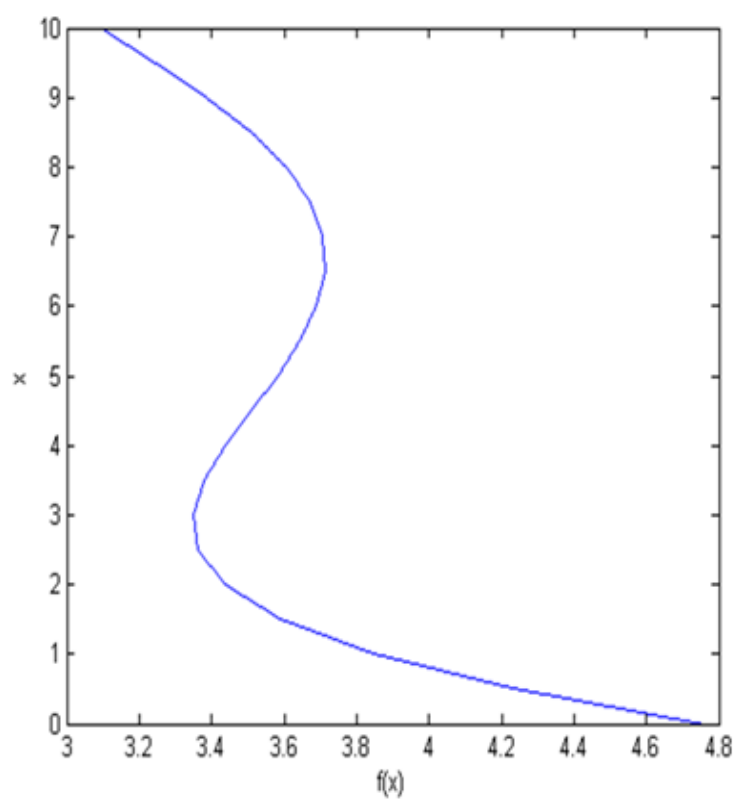

Fig 3: Spine shape drawn for table 1 values

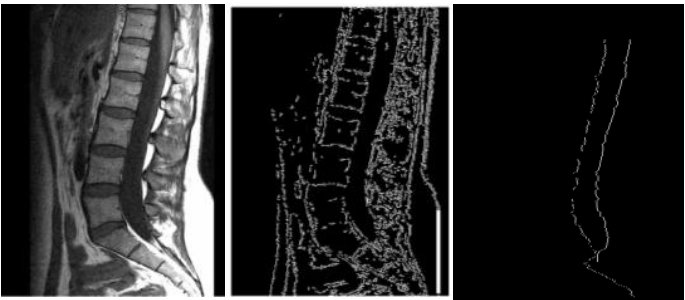

Fig 4: a) MRI input Image b) Sobel edge algorithm result c) Detected spinal canal

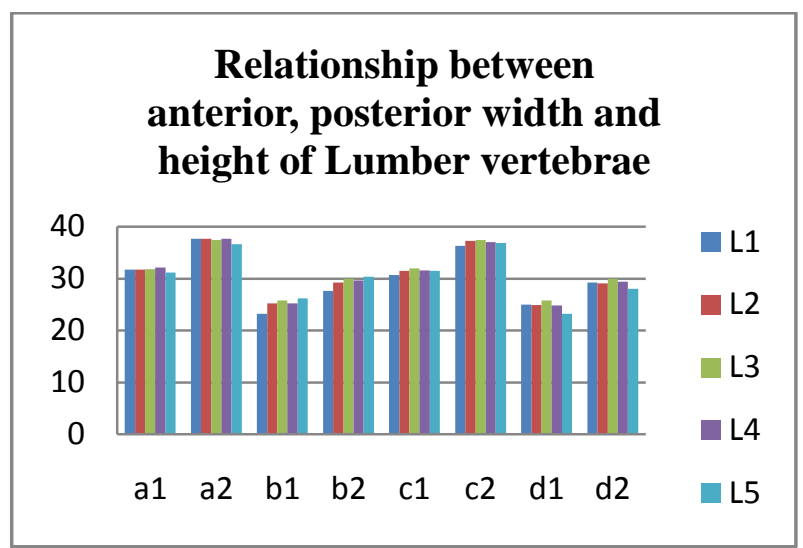

Fig 5: Relation between vertebra anterior, posterior width and height of Lumber vertebrae L1 to L5

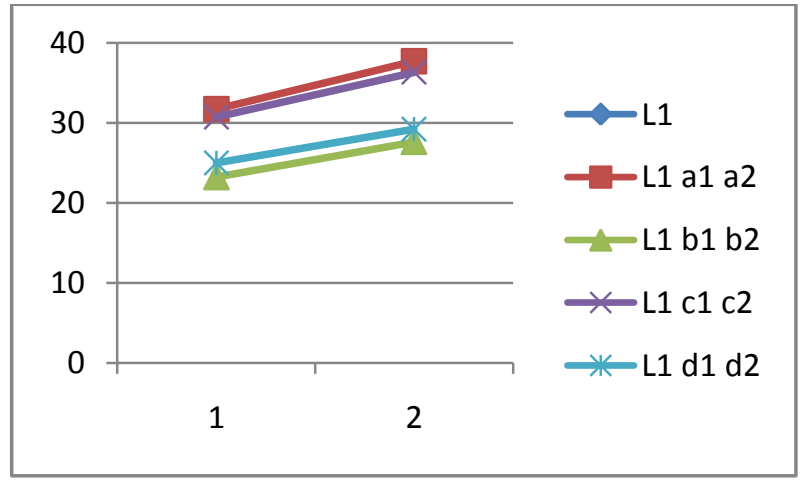

Fig 6: Relation between range of values of vertebra anterior, posterior width and height of Lumber vertebra L1

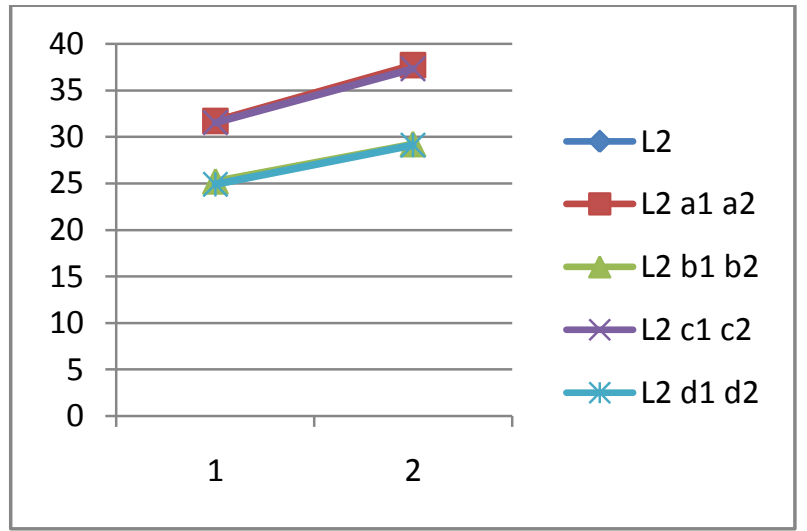

Fig. 7 Relation between range of values of vertebra anterior, posterior width and height of Lumber vertebra L2 


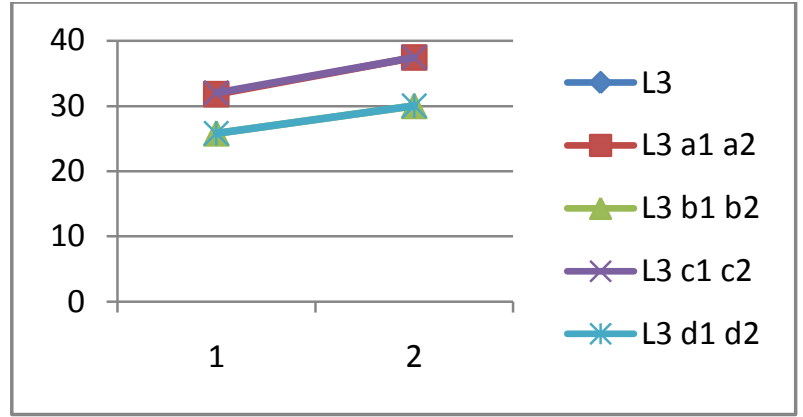

Fig. 8 Relation between range of values of vertebra anterior, posterior width and height of Lumber vertebra L3

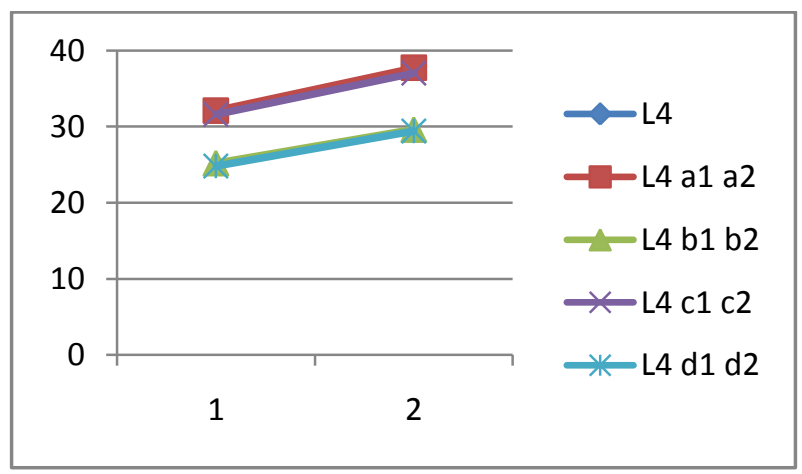

Fig. 9 Relation between range of values of vertebra anterior, posterior width and height of Lumber vertebra L4

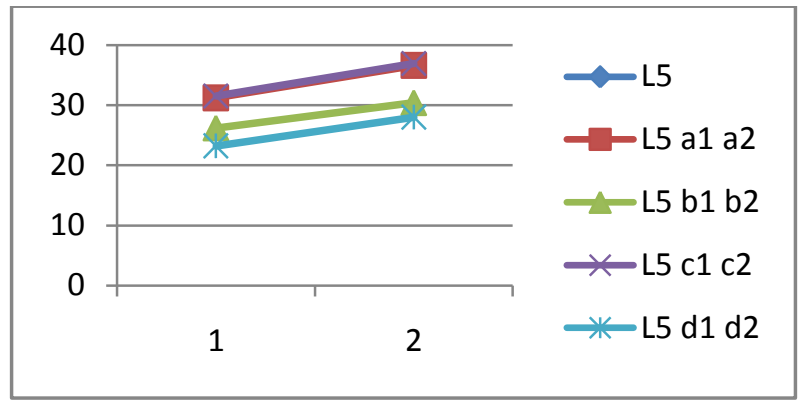

Fig. 10 Relation between range of values of vertebra anterior, posterior width and height of Lumber vertebra L5

The following relations are derived between vertebrae anterior width A, height $\mathrm{B}$ and posterior width $\mathrm{C}$, height $\mathrm{D}$ from above results. The relations are given in Table 2.

Table 2. Relation between statistical properties

\begin{tabular}{|c|c|c|}
\hline S.NO & Vertebra Name & Derived Relation \\
\hline 1 & L1 & B $<$ A,C C $\sim=A, D<A, C$ \\
\hline 2 & L2 & B $\sim=\mathrm{D}, \mathrm{A} \sim=\mathrm{C}, \mathrm{B}, \mathrm{D}<\mathrm{C}, \mathrm{D}$ \\
\hline 3 & $\mathrm{~L} 3$ & $\mathrm{~B}, \mathrm{D}<\mathrm{A}, \mathrm{C}, \mathrm{B}=\mathrm{D}, \mathrm{C}<\mathrm{A}$ \\
\hline 4 & $\mathrm{~L} 4$ & $\mathrm{~B}, \mathrm{D}<\mathrm{A}, \mathrm{C}, \mathrm{A} \sim=\mathrm{C}, \mathrm{B} \sim=\mathrm{D}$ \\
\hline 5 & $\mathrm{~L} 5$ & $\mathrm{~B}, \mathrm{D}<\mathrm{A}, \mathrm{C}, \mathrm{C}=\mathrm{A}$ \\
\hline
\end{tabular}

In lumber region width of vertebrae greater than the height. In lumber region anterior height and posterior heights are almost equal, similarly anterior and posterior widths are also almost equal. Based on relations derived between A,B,C, and D, vertebrae are labelled with $96 \%$ accuracy. The values are validated by the Ortho Domain expert.

\section{CONCLUSIONS}

A novel method VESTAL is proposed to label lumber vertebrae and inter vertebral discs. The proposed method uses statistical properties and produces $96 \%$ accuracy in labelling vertebral and intervertebral disc. This labelling is very useful for detecting degenerated disc. In future this work can be extended for detecting abnormal region and to classify the degenerated discs.

\section{REFERENCES}

[1] R. S. Alomari, J. J. Corso, and V. Chaudhary, 2011 "Labelling of lumbar discs using both pixel and object level features with a two-level probabilistic model," IEEE Trans. Med. Imag., vol. 30, no. 1, pp. 1-10, (Jan. 2011

[2] I.Gilad and M.Nissan, 1985. "Sagittal evaluation of elemental geometrical dimensions of human vertebrae" J.Anat.,143,115-120.

[3] Sofia K. Michopoulou et al., 2009. "Atlas Based Segmentation of Degenerated Lumbar Intervertebral Discs From MR Images of the Spine" IEEE Trans. Biomed. Eng., vol. 56,no.9, pp. 2225-2231, (Sept. 2009).

[4] S.H. Huang, Y.H. Chu, S.H. Lai, and C. L. Novak, 2009. "Learning-based vertebra detection and iterative normalized cut segmentation for spinal MRI," IEEE Trans. Med. Imag., vol. 28, no. 10, pp. 1595-1605

[5] S. Seifert, I. Wachter, G. Schmelzle, and R. Dillmann, 2009. "A knowledge based approach to soft tissue reconstruction of the cervical spine," IEEE Trans. Med. Imag., vol. 28, no. 4, pp. 494-507, (Apr. 2009).

[6] Ayse Betul Oktayand Yusuf Sinan Akgul, 2013. "Simultaneous Localization of Lumbar Vertebrae and Intervertebral Discs With SVM-Based MRF" IEEE Transaction on Biomedical Engineering Vol 60, No 9,

[7] Ruiqiong Shi ,Dongmei Sun, Zhengding Qiu and Kenneth L.Weiss, 2007. "An efficient method for Segmentation of MRI Spine Images" IEEE 2007, 14244-1078-9/07

[8] Mohammed Benjelloun, Sard Mahmoudi, and Fabian Lecron, "AFramework of Vertebra Segmentation Using the Active Shape Model-Based Approach" Hindawi Publishing Corporation, International Journal of Biomedical Imaging, Volume 2011, Article ID 621905, 14 pages

[9] Z. Peng, J. Zhong, W. Wee, and J. Lee, 2005 "Automated vertebra detection and segmentation from the whole spine MR images," in Proc. Conf. IEEE Eng. Med. Biol. Soc., (Jan. 2005), vol. 3, pp. 2527-2530.

[10] S. Schmidt, J. Kappes, M. Bergtholdt, V. Pekar, S. Dries, D. Bystrov, and C. Schnorr, 2007. "Spine detection and labelling using a parts-based graphical model," in Proc. 20th Int. Conf. Inf. Process. Med. Imag., vol. 4584,pp. $122-133$ 
[11] Y. Zheng, M. Nixon, and R. Allen, 2004. "Automated segmentation of lumbar vertebrae in digital video fluoroscopic images," IEEE Trans. Med. Imag., vol. 23, no. 1, pp. 45-52, (Jan. 2004).

[12] J. J. Corso, R. S. Alomari, and V. Chaudhary,2008. "Lumbar disc localization and labelling with a probabilistic model on both pixel and object features,"in Proc. Med. Image Comput. Assisted Intervention Conf., vol. 52, pp. 202-210.

[13] David Major ,Jiri Hladuvka, Florian Schulze, Katja Buhler, 2013. " Automated landmarking and labelling of fully and partially scanned spinal columns in CT images" Elsevier :Medical Image Analysis 17, 11511163

[14] Andreas Tunset, Per Kjaer, Shadi Samir Chreiteh and Tue Secher Jensen, 2013. "A method for Quantitative measurement for lumber intervertebral disc structure: an intra-inter rater agreement and reliability study” vol 21.
[15] C. Bhole, S. Kompalli, and V. Chaudhary, 2009. "Context-sensitive labelling of spinal structures in MRI images," in Proc. SPIE Med. Imag., pp. 803-806

[16] S. Ghebreab and A. W. M. Smeulders, 2004. "Combining strings and necklaces for interactive three dimensional segmentation of spinal images using an integral deformable spine model," IEEE Trans. Biomed. Eng., vol. 51,no.10, pp. 1821-1829, (Oct. 2004).

[17] B. Glocker, J. Feulner, A. Criminisi, D. R. Haynor, and E. Konukoglu, 2012. "Automatic localization and identification of vertebrae in arbitrary field of view CT scans," in Proc. Med. Image Computing and. Computer Assisted Intervention Conf., vol. 3, pp. 590-598.

[18] L. R. Long and G. R. Thoma, 2000. "Use of shape models to search digitized Spine X-rays," in Proc. 13th IEEE Symp.Comput.Based Med. Syst.,Washington, DC, USA, pp. 255-260. 